\title{
The Theatre methodology for facilitating discussion in human- robot interaction on information disclosure in a home environment
}

\author{
Dag Sverre Syrdal, Kerstin Dautenhahn, Michael L. Walters, Kheng Lee Koay, Nuno R. Otero
}

\begin{abstract}
Our research is concerned with developing scenarios for robot home companions as part of the EU project LIREC. In this work, we employed a particular methodology to gain user feedback in early stages of robot prototyping: the Theatre HRI (THRI) methodology which we have recently introduced in a pilot study. Extending this work, this study used a theatre presentation to convey the user experience of domestic service robots to a group of participants and to gain their feedback in order to further refine our scenarios. The play was designed both from the perspective of projected technological development of the LIREC project, as well as for facilitating engagement with an audience of secondary school students. At the end of the play the audience was involved in a discussion regarding issues such as acceptability of the scenario and the intrahousehold disclosure of information by the robot. Findings suggest that this methodology was effective in eliciting discussion with the audience and that problems related to intra-household disclosure of information were best resolved by clear-cut solutions tied to ownership and clear principles.
\end{abstract}

\section{INTRODUCTION}

O ONE of the major challenges when prototyping interactions with emergent and future robotic technologies is the difficulty in situating the possible interactions within a context that can be translated to the everyday experience of the prospective user. This issue is compounded by the highly dynamic, subjective nature of such experiences and the equally complex manner that the technologies may impact them [1]. Some technologies however may not have the stability required to function in an effective and safe manner autonomously for sustained periods of time outside of constrained settings. This creates difficulties in prototyping them for their intended setting and use. Dautenhahn [2] notes that these issues pose particular problems for the field of Human-Robot Interaction (HRI) as a whole, as well as necessitating a high degree of pragmatism and creativity when developing methodologies to examine how prospective users reason, feel or interact with these technologies. Also, live interactions with robotic technology in complex

Manuscript received March 1, 2011 This work was conducted within the European FP7 ICT-215554 project LIREC (LIving with Robots and Interactive Companions)

Dag Sverre Syrdal, Kerstin Dautenhahn, Kheng Lee Koay, Michael and Leonard Walters are with the Adaptive Systems Research Group, School of Computer Science, University of Hertfordshire, Hatfield, UK. (e-mail: \{d.s.syrdal; k.dautenhahn; k.l.koay; m.l.walters\} @ herts.ac.uk). Nuno R. Otero is with the Department of Information Systems University of Minho, Guimarães, Portugal (e-mail: nuno.otero@dsi.uminho.pt). scenarios can often only involve a relatively small number of participants, due to the cost, in time and resources to setup and run of the experiments. While it is often desirable to run studies with a number of participants as large as possible, this need must be balanced with the ability to create rich avenues of interaction as well as datacapture for subsequent analysis.

\section{A. The Use of Scenarios}

The referred to constraints emerging from the specificities of HRI lead us to the use of scenario-based methods of user-studies taken from the related field of Human-Computer Interaction (HCI). Scenarios are stories created about the users and their interactions with artefacts in a specific context (see, for example, Carroll [3], [4]). While these methods were initially conceived as an aid for the designer of such technologies as they allow for the exploration and focus of issues which otherwise may not have been available to the designers [3] they also allow the researcher a greater ability to convey some of the salient issues to the prospective user in a way which pure descriptions of the technology may not. As Carroll [3] points out this becomes a way "to anchor design discussion" with other stakeholders in the design process.

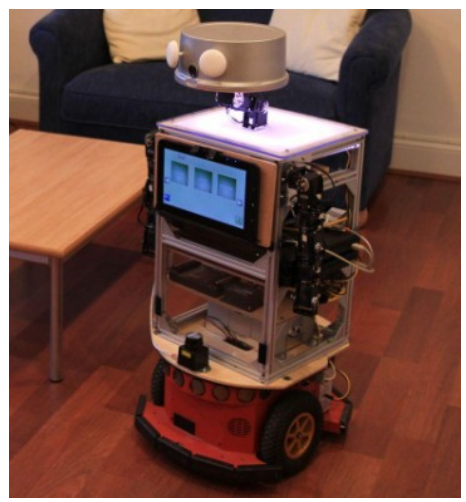

Figure 1 Sunflower - A modified Pioneer robot - note background is not from the theatre production

Scenarios can be instantiated in a variety of forms: written stories, with or without suggestive illustrations, animations, video or dramatizations. In HRI, the use of video-based instantiations of scenarios have been used extensively in user-studies. Some of these studies have focused on studies on particular issues like responses to specific robot appearances [5] spatial behavior [6], as well as interactional styles [7], [8]. It has also been used for more general explorations of user requirements and reasoning about the use of a robot within domestic settings [7], [9], [10]. In the above examples, researchers had the 
opportunity to elicit feedback on specific proposed technologies as well as been able to explore more general issues related to the issues raised by these technologies.

\section{B. Theatre Methodology}

The use of theatre and drama techniques to instantiate scenarios, in order to explore issues surrounding emergent and future technologies, has been explored in HumanComputer Interaction as a method for prompting discussion between designers and stakeholders to as well as aiding in the development process [11-15] In this sense, theatre can be considered as a means of prototyping, along with a higher fidelity than that of storyboarding or video, as it adds a sense of physical presence and real-time performance, which is seen as an important facet of HRI [16], and is easily lost when seen through the screen, or through a second-hand written account. It is also costeffective, in that it addresses design-issues at an earlier stage than live interactions, where much of the technical development needs to have been locked in place. As such, it offers a possible middle-ground between the speed of video mock-ups and the depth of live human-robot interactions. Also, by allowing actors to interact with live robots, they can respond to the audience's reactions and improvise (limited to some extent by the robots' capabilities) to enhance the production of the presentation.

\section{Study Context}

The focus of this study is technology developed within the FP7 Integrated project, LIREC (Living wIth Robots and intEractive Companions). Specifically, this study is concerned with some of the issues raised in the University of Hertfordshire Robot Home Companion Scenario. This scenario posits an agent that is capable of migrating between different physical embodiments (described in [17] and [18]). Migration here means that the agent's 'mind' (e.g. memory, reasoning etc.) can move between different robots. This agent has two robotic embodiments in this particular scenario; a modified Pioneer embodiment $^{1}$ called Sunflower (see fig 1) and a Sony AIBO embodiment, i.e. a small zoomorphic (dog-like) robot.

. The functions it provides for its user is that of a cognitive prosthetic (e.g. reminder function) [19], a fetch-and-carry function [20], as well as a game to enhance remote communication with another person (social mediator, described in [21] and shown in figure 3).

Previously a pilot study was conducted, in order to investigate the general feasibility of this methodology for HRI research and the findings are reported in Chatley et al. [22]. This pilot study used a narrative centered around a single elderly user, and focused on his use of the agent as both a cognitive prosthetic and fetch-and-carry aid. While the overall experience of using this methodology was positive, it was felt that the narrative was possibly not

\footnotetext{
1 Commercially available from Mobilerobots. http://www.mobilerobots.com/
}

engaging enough for the audience, and that the post-play audience discussion tended to focus on aspects of the agent that were not of primary interest in the development of the University of Hertfordshire Showcase Scenario. The clearest example of this was a strong focus of the discussion on various aspects of robot's speech, which was not the main area of interest for the study (we are not using speech recognition in the Robot House showcase). Also, the pilot study used only a small audience size so the conclusions drawn from the study were limited.

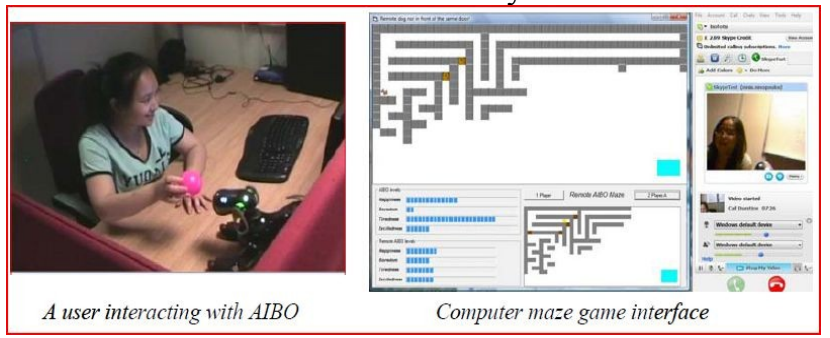

Figure 2 Social Mediator Game - Note photo not taken from the theatre study.

\section{II.DEVELOPMENT OF THE SCRIPT}

For this study we decided to target an audience of secondary school children who are generally very versatile and interested in the use of new computer technology, and will most likely be the generation who might in future benefit from the actual deployment of multi-purpose robot home companions. Based on these and the above considerations described in section I, the goals for this particular study were as follows:

1. To portray a scenario based on the technologies developed in the LIREC project.

2. To create a more engaging narrative and introduce dramatic tension.

3. To make the narrative appropriate for an audience of secondary school students.

4. To focus the discussion towards topics of interest of the LIREC project.

a. The acceptability of the scenario

b. Issues surrounding privacy

The first goal was achieved by making the narrative of the story rely heavily on the technologies developed in the LIREC project, allowing the cognitive prosthetic, fetchand-carry and migration capabilities of the agent to feature heavily in the plot.

In order to meet the second and third goal, the researchers decided to introduce a second character, the grandson of the primary user. Not only would this allow the younger audience a greater chance for mutual identification with one of the characters in the play, but it would also allow the narrative to exhibit more tension as the goals of the two characters could conflict. The fourth goal was met by both creating a narrative which raised particular questions, as well as by giving the participants a brief questionnaire at the end of the play to get them to consider specific issues individually before the plenary discussion. To avoid any misunderstandings regarding speech (which dominated the discussions with the 
audience in our previous pilot study [22]), it was decided to explicitly show the characters using a touch-screen interface to interact with the robot.

It was decided to base the dramatic conflict around the issues of privacy and ownership of the agent. The issue of information retention of such an agent has been considered within the LIREC project in Vargas et al. [23], and we have previously raised privacy as a potential issue in domestic service robots [24]. Interestingly, however, Sharkey and Sharkey [25] highlights this as a particular problem within a household, especially in relations to minors in a household and their carers. As such, it is of interest to HRI in general and the LIREC project in particular. Also, issues of parental control and monitoring of technology usage is a common area of conflict in families with teenagers [26], which suggested that this particular conflict would resonate with the audience.

\section{OVERVIEW OF THE PLOT}

\section{A. The Characters:}

\section{1) David}

David is a single (most likely widowed, but this was not addressed in the play), retired man in his mid-to late $60 \mathrm{~s}$. While physically and mentally fit and fairly adept with technology, he has acquired a "Companion" to help him with various aspects of his everyday life. He has children and grandchildren that he keeps in touch with, via Skype and email.

\section{2) The Grandson}

The unnamed grandson is in his late teens, and has come to live with David for a semester. Like most teenagers, he is curious about new technologies, especially the possibilities for leisure activities they provide. He has a close relationship with his grandfather, and they often joke and tease each other. He also enjoys doing things with his younger brother, in particular computer console games.

\section{3) The Companion}

The Companion is a migrating agent which can be embodied in several platforms. Its main embodiment is that of the Sunflower robot (see picture in fig 1), but it is also embodied in an AIBO in the play for the purposes of playing the social mediator game. Other embodiments are referred to as possibilities in the play, but the agent does not use them. The primary function of the agent is that of a cognitive prosthetic, and its secondary functions are that of mediating communications through notifications of received emails and text messages, and fetch-and-carry assistance. The agent is also capable of playing a social mediator game, and this is demonstrated in the play.

\section{B. Location}

The entirety of the plot takes place in one location, David's home. The stage was set up to convey the impression of three distinct areas. A parlour area to sit and relax, a dining area and a work area with a laptop. There were two entrances and exits to the stage, one to the outside world, and one to the bedrooms and the remainder of the home. See fig 3 for an overview of the stage area.

\section{Scene-by-scene overview}

\section{1) Scene 1: The Grandson Arrives}

In this scene, the grandson arrives at the home. He is startled by the robotic embodiment of the agent. The agent is introduced as well as its functions and embodiments. Migration from the Sunflower embodiment to the AIBO embodiment is demonstrated. The grandson suggests that he could use the agent to play with his brother, but David does not seem keen to let his grandson use it.

2) Scene 2: The Message from the School This scene takes place later in the same week as the

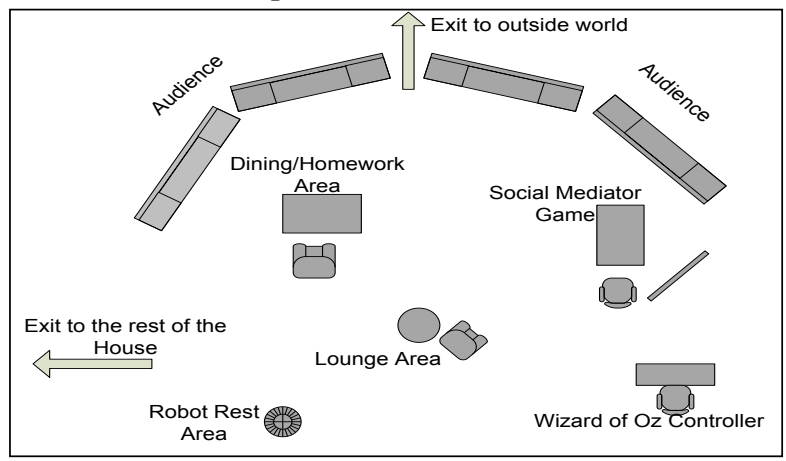

Figure 3 Layout of the Stage Area previous scene. While the grandson is away, David receives a message through the agent regarding a schedule change in the school. The grandson returns as scheduled and is greeted by the robot, which now recognizes him as a member of the household

\section{3) Scene 3: Fetch and Carry}

This scene takes place on the next weekend. The grandson is doing homework and uses the fetch-and-carry functionality to get one of David's books. There is a brief interchange where David expresses some misgivings about his grandson using the agent.

4) Scene 4: Social Mediator

This scene takes place about a week after the previous scene. The grandson is using the agent in its AIBO embodiment to play the social mediator game remotely with his little brother and reveals that he has used the agent to organize a surprise birthday party for David. David arrives home, and needs the agent to help carrying some washing. The agent migrates from the AIBO embodiment into the Sunflower embodiment. The play ends with David asking the robot to tell him what his grandson has been up to.

\section{DELIVERY}

\section{A. Audience and Setting}

The audience was a group 46 secondary school students aged 13-14. They were evenly spread in gender and had been specifically selected by their grade teachers for participating in a subsequent robotics workshop. 
The play took place in the assembly hall of the participant's school as part of a day focused on robotics. The students were seated in a horse-shoe shape around a central area in which the play took place. It was hoped that having the movement of the actors and the robots taking place in the same space, and on the same level as the audience, would allow for a greater appreciation of the physical aspects of the interaction with the robot.

\section{B. Procedure}

After the audience was seated, the play was introduced by one of the researchers, and it was explicitly stated that the researchers would like to discuss some of the issues in the play with the students afterwards. During the play, the robots were controlled by members of the research team, using the Wizard of Oz (WoZ) [27] method (unknown to the audience but revealed during the discussion). After the play, a brief questionnaire was given to the students. The questionnaire was kept deliberately simple and included three Yes/No questions:

1. Should the robot tell the grandfather what his grandson has been doing?

2. Was the robot useful?

3. Would you want to live in a house that had a robot like this?

The questions were intended to both provoke the participants into thinking about issues raised in the play as well a source of data capture. After completing the questionnaire, the participants discussed their answer with each other and the researchers. The researchers guided this discussion by referring back to the questions in the questionnaire, and asking the participants as to the reasoning behind their answers. This discussion was recorded using a video-camera and microphones.

\section{V.RESULTS}

\section{A. Questionnaire responses}

As stated above, there were two strands of interest to the researchers. The first was the acceptability of the robot and the scenario as a whole, and the second was how the participants engaged with the scenario in terms of what course of action the robot should take.

\section{1) Acceptability of the robot and its use in the} scenario

A significant majority of participants stated in the questionnaires that the robot was useful within the scenario (39 out of $\left.46, \chi^{2}(1)=22.26, p<.001\right)$ and that they would want to live in a house with a robot with the capabilities displayed in the play (35 out of 46, $\left.\chi^{2}(1)=12.52, p<.00\right)$. This suggests that the participants to a large extent accepted the scenario as presented in the play and also saw the use for the robot in their everyday lives.

\section{2) Information Disclosure}

In terms of whether or not the robot should let the owner of the robot know what his grandson had been doing, 31 students stated in their questionnaires that the robot should reveal this information, while 15 stated that it should not. Thus there was a significant majority $\left(\chi^{2}(1)=5.57, p=.018\right)$ in favour of this course of the robot disclosing this information.

\section{B. Subsequent Discussion}

\section{1) Acceptability of the robot and its use in the scenario}

The participant's responses regarding the acceptability of the robot and its use within the scenario primarily consisted of the participants' referring back to instances in the play where the two characters in the play had found a use for the robot. The main benefit referenced in the discussion was the cognitive prosthetic function, in particular for reminding the user of social appointments and school assignments. The fetch-and-carry function was not referred to without probing from the researcher leading the discussion, and statements following this probe focused on how the participants did not feel a need for this particular function. When discussing the responses to the second question, whether or not participants would want a robot like this, participants were less focused on the use-value of the robot as displayed in the scenario than the novelty of the robot and the possibility of uses not shown in the play.

\section{2) Information Disclosure}

While, as noted above, there was a majority in favour of the robot disclosing details about the grandson's actions to the robot's owner, there was a sizeable minority which did not want the robot to take this course of action. The analysis of the reasoning behind these answers was descriptive and intended to bring out the breadth of reasoning rather than quantifying the prevalence of them within the sample, as not every single participant gave their reasoning in the subsequent discussion. The types of arguments were classified in two stages, the first was to classify them in regards to whether or not they were in favour of disclosure, the second was dividing them into types of reasoning.

\section{a) Reasoning against disclosure}

There were two main lines of argument against the robot disclosing the information to the owner. The first argument was based on the principle that the grandson had a right to privacy, irrespective of the ownership of the robot. The following statement is an example of this argument :

"I don't think it is right that he could see what his grandson was doing just because it is his robot. I wouldn't want my parents to keep an eye on me like that." The second line of reasoning was tied more closely to the intention of the grandson in using the computer. The fact that the grandson was using the robot for something that was going to be a nice surprise for the owner, meant that it was not right for the robot to reveal the information. This is shown in statements like this: 
"...after all, the robot would ruin the surprise for David (the owner) if he lets him know about it, so I didn't think it should tell."

\section{b) Reasoning for disclosure}

There were several different reasons for the robot revealing the information. One of the most repeated reasons was that of ownership. As the robot was, in fact, owned by somebody else, the grandson should not expect it to keep information from him. Also, the issue of 'loyalty' was highlighted.

"It is the grandfather's robot. It shouldn't keep secrets from him."

"It answers to David (the owner), it would be wrong to not tell him."

Some participants focused on the grandson's deception of the owner. While similar to the loyalty argument, the focus was on the grandson's obligations rather than that of the robot's. Here the argument was that the robot should tell the owner as the grandson was wrong to deceive him.

"I know that he was nice and wanted to give his grandfather a surprise, but he still lied to him. I think the robot should tell David about it."

The final line of argument, however, centered around the participants being unsure if the robot could or should be able to distinguish between information that it is unproblematic to share and information that is sensitive.

"...then how would the robot know that a surprise birthday party is a good thing? How does it know what a birthday party is?"

"I don't think that the robot should decide what to tell and what not to tell. I don't think a robot should do that sort of thing."

\section{DISCUSSION}

\section{A. Overall Comments on the Discussion}

It seems as if the changes and refinements to the theatre methodology for HRI did manage to fulfill the aims set forth in section II. We were able to create a scenario instantiation which the audience could engage with and the addition of the questionnaire served to focus the discussion towards topics which were of direct interest for the development of the technologies within the LIREC project.

\section{B. Scenario Acceptability}

The results from the questionnaire and the discussion clearly suggests that the participants were able to relate to the scenario as portrayed in the play and accepted the robot and its use within the scenario. The participants referred back to functions within the scenario as potentially useful and expressed an interest in living in households which had robots with these capabilities. They also volunteered tasks they could see the robot helping them with. Not surprisingly, the fetch-and-carry functionality of the robot was not a key topic for the audience. This could be explained by their age and that they probably have not had significant encounters yet of being mobility impaired through accident, illness, or old age. However, the audience accepted such a functionality as part of the companion robot's services. This is encouraging, since it shows that the demonstrated functionalities of a multi-purpose companion home robot, as we are aiming to develop, were overall acceptable, although not all functionalities were of high utility to the audience. A future home companion robot will have to be acceptable not only to their primary user, but also to secondary users such as family members.

\section{Disclosure}

Despite the clear majority in favour of disclosure, the division within the audience suggests that this is still an issue that should be addressed and as such echoes the sentiment of Sharkey \& Sharkey [22] namely that issues of intra-household disclosure are potentially problematic. The discussion, however, did point to several ways of mitigating these issues.

Firstly, the focus on ownership is a potential way of mediating this issue. If the ownership and control is clearly communicated to the users, this might reduce the self-disclosure of the (secondary) users to the agent and so reduce the threats to their privacy. One way of achieving this might be through the way suggested in [28]. where robotic embodiments clearly display the identity of the user whose agents is currently using this embodiment. This may not be sufficient though, especially if the agent is constantly performing its duties throughout the household, making it difficult to avoid.

The arguments based on ethical reasoning are also quite interesting, as they highlight not only the conflict between principles such as principal rights to privacy and the problems of deception, but also factors such as the intent of the human users.

Taken together they create a complex ethical field to navigate. To address these argument appropriately, an 'ethical agent' will have to recognise and weigh these concerns against each other in each individual case in order to reach decisions that are acceptable to both primary and secondary users. Interestingly, one of the case studies in Anderson and Anderson's [29] machine ethics was the decision to disclose information to a third party.

However, this approach may meet some resistance from prospective users echoing the final concerns of the participants. Resistance to the robot making such a decision is here either based on the inability of the robot to be able to make an informed ethical decision (similar Sharkey's [30] argument concerning decision-making for military robots) or a more general opposition to the robot making ethical decisions on behalf of humans. Such an opposition to a robot's decision making is not uncommon, and has been suggested in surveys such as Takayami et al. [31]. Empowering the individual user to mark certain information as not suitable for disclosure might be a better option, similar to the features offered for some web browsers described in [32]. To conclude, the issues of 
intra-household disclosure of information were problematic to this audience. However, it seems that rather than exclusively relying on technological advances to resolve them, parts of this audience preferred more clear-cut solutions tied to ownership and clear principles. The breadth of responses collected in this study will be useful for our further work on these issues, allowing us to create quantitative questionnaires encompassing the possible dimensions of reasoning that underlies attitudes towards such disclosure for use either in surveys or as part of live interactions study.

\section{Conclusions and Future Work}

This study represents a further refinement of the theatre methodology for HRI, and suggests that this methodology is an effective way to prototype interactions and open up avenues of discussions with prospective users of such technologies. We are currently exploring several avenues to develop this methodology for HRI studies. An attractive direction is to tie particular questions to particular scenes, highlighting more specific facets of interactions. We intend to continue this work, in particular in the area of ways to further facilitate discussion with different audiences. Generally, this work demonstrates that the Theatre HRI methodology can be used in early stages of the development of fully autonomous robots operating in complex scenarios in order to gain user feedback.

\section{REFERENCES}

[1] M. Buchenau and J. F. Suri, "Experience prototyping," in Proceedings of the conference on Designing interactive systems processes, practices, methods, and techniques - DIS '00, New York City, New York, United States, 2000, pp. 424-433.

[2] K. Dautenhahn, "Methodologies and Themes of Human-Robot Interaction - A Growing Research Field," International Journal of Advanced Robotics Systems, vol. 4, no. 1, pp. 103-108, 2007.

[3] J. Carroll, "Five reasons for scenario-based design," Interacting With Computers, vol. 13, pp. 43-60, 2000.

[4] J. Carroll, Scenario-based Design. Envisioning Work and Technology in System Development. New York: John Wiley \& Sons, 1995.

[5] M. L. Walters, D. S. Syrdal, K. Dautenhahn, R. te Boekhorst, and K. L. Koay, "Avoiding the uncanny valley: robot appearance, personality and consistency of behavior in an attention-seeking home scenario for a robot companion," Autonomous Robots, vol. 24, no. 2, pp. 159-178, 2007.

[6] S. Woods, M. Walters, K. L. Koay, and K. Dautenhahn, "Comparing human robot interaction scenarios using live and video based methods: towards a novel methodological approach," in 9th IEEE International Workshop on Advanced Motion Control, 2006., Istanbul, Turkey, 2006, pp. 750-755.

[7] D. S. Syrdal, K. L. Koay, M. Gacsi, M. L. Walters, and K. Dautenhahn, "Video prototyping of dog-inspired non-verbal affective communication for an appearance constrained robot," in 19th International Symposium in Robot and Human Interactive Communication, Viareggio, Italy, 2010, pp. 632-637.

[8] M. Lohse et al., "Evaluating extrovert and introvert behaviour of a domestic robot \&\#x2014; a video study," in RO-MAN 2008 - The 17th IEEE International Symposium on Robot and Human Interactive Communication, Munich, Germany, 2008, pp. 488-493.

[9] A. Cesta, G. Cortellessa, M. V. Giuliani, F. Pecora, M. Scopelliti, and L. Tiberio, "Psychological Implications of Domestic Assistive Technology for the Elderly," PsychNology Journal, vol. 5, no. 3, pp. 229-252, 2007.

[10] D. S. Syrdal, N. Otero, and K. Dautenhahn, "Video prototyping in humanrobot interaction," in Proceedings of the 15th European conference on Cognitive ergonomics the ergonomics of cool interaction - ECCE '08, Funchal, Portugal, 2008, p. 1.

[11] A. F. Newell, A. Carmichael, M. Morgan, and A. Dickinson, "The use of theatre in requirements gathering and usability studies," Interacting With Computers, vol. 18, no. 5, pp. 996-1011, 2006.
[12] A. F. Newell, M. E. Morgan, P. Gregor, and A. Carmichael, "Theatre as an intermediary between users and CHI designers," in CHI '06 extended abstracts on Human factors in computing systems - CHI '06, Montr\&\#233;al, Qu\&\#233; bec, Canada, 2006, p. 111

[13] A. Light, L. Weaver, P. Healey, and G. Simpson, "Adventuresin the Not Quite Yet: using performance techniques to raise designawareness about digital networks.," Undisciplined! Design Research Society Conference 2008, Sheffield Hallam University, Sheffield, UK, 16-19 July 2008, 2008.

[14] G. Iacuccui and K. Kuuti, "Everyday Life as a Stage in Creating and Performing Scenarios for Wireless Devices," Personal and Ubiquitous Computing, vol. 6, pp. 299-306, 2002.

[15] T. Salvador and K. Howells, "Focus troupe," in CHI 98 conference summary on Human factors in computing systems - CHI '98, Los Angeles, California, United States, 1998, pp. 251-252.

[16] J. Wainer, D. Feil-seifer, D. Shell, and M. Mataric, "The role of physical embodiment in human-robot interaction," in ROMAN 2006 - The 15th IEEE International Symposium on Robot and Human Interactive Communication, Univ. of Hertfordshire, Hatfield, UK, 2006, pp. 117-122.

[17] LIREC, "Deliverable D 8.1 Foundations of Migrating Companions Available through http://lirec.eu." 2009.

[18] D. S. Syrdal, K. L. Koay, M. L. Walters, and K. Dautenhahn, "The boyrobot should bark! - Children's Impressions of Agent Migration into Diverse Embodiments," in Proceedings New Frontiers in Human-Robot Interaction, a symposium at the AISB2009 Convention, Heriot Watt University, Edinburgh, Scotland, 2009.

[19] J. Modayil, R. Levinson, C. Harman, D. Halper, and H. Kautz, "Integrating Sensing and Cueing for More Effective Activity Reminders," in AAAI Fall 2008 Symposium "AI in Eldercare: New Solutions to Old Problems" November 7-9, Washington, DC, USA, , pp. 60-66.

[20] H. Huettenrauch and K. Severinson Eklundh, "Fetch-and-carry with CERO: Observations from a long-term user study with a service robot.," Proceeding of the 11th IEEE International Workshop on Robot and Human Interactive Interactive Communication(RO-MAN 2001), Berlin, Germany Sept 25-27, 2002, pp. 158-163, 2002.

[21] F. Papadopoulos, K. Dautenhahn, W. C. Ho, and M. L. Walters, "AIBOCOM: Designing robot enhanced human-human remote communication technology," in Proceedings of the Kansei Engineering and Emotion Research International Conference 2010 (KEER2010), 2-4 March 2010, Arts et Metiers ParisTech, Paris, France, 2010, pp. 671-682.

[22] A. R. Chatley, K. Dautenhahn, M. L. Walters, D. S. Syrdal, and B. Christianson, "Theatre as a Discussion Tool in Human-Robot Interaction Experiments - A Pilot Study," in 2010 Third International Conference on Advances in Computer-Human Interactions, St Maarten, Netherlands Antilles, 2010, pp. 73-78.

[23] P. A. Vargas, W. C. Ho, M. Lim, S. Enz, Y. Fernaeus, and R. Aylett, "To Forget or Not to Forget: Towards a Roboethical Memory Control," in Proceedings Killer Robots or Friendly Fridges a symposium at the AISB2009 Convention, Heriott-Watt University, Edinburgh, Scotland, 2009.

[24] D. S. Syrdal, M. L. Walters, N. R. Otero, K. L. Koay, and K. Dautenhahn, "He Knows When You Are Sleeping - Privacy and the Personal Robot," in Proccedings of AAAI-07 Workshop W06 on Human Implications of Human-Robot Interaction, Vancouver, British Columbia, Canada, 2007, pp. 28-33.

[25] N. Sharkey and A. Sharkey, "The crying shame of robot nannies: An ethical appraisal," Interaction Studies, vol. 11, no. 2, pp. 161-190, 2010

[26] R. Wang, S. M. Bianchi, and S. B. Raley, "Teenagers' Internet Use and Family Rules: A Research Note," Journal of Marriage and Family, vol. 67 , no. 5, pp. 1249-1258, 2005.

[27] A. Green, H. Huttenrauch, and K. Severinson Eklundh, "Applying the Wizard-of-OZ Framework to Cooperative Service. Discovery and Configuration," Proc. 2004 International Workshop on Robot and Human Interactive Interactive Communication., pp. 575-580, Sep. 2004.

[28] K. L. Koay, D. S. Syrdal, K. Dautenhahn, K. Arent, L. Malek, and B. Kreczmer, "Companion Migration - Initial Participants' Feedback from a Video-Based Prototyping Study," Mixed Reality and Human-Robot Interaction Wang, Xiangyu (Ed.), vol. 47, pp. 133-151, 2011.

[29] S. L. Anderson and M. Anderson, "EthEl: Toward a Principled Ethical Eldercare robot," in Robotic Helpers: User Interaction, Interfaces, and Companions in Assistive and Therapy Robotics, Workshop at HRI'08, 3rd ACM/IEEE International Conference on Human-Robot Interaction, Amsterdam, The Netherlands, 2008.

[30] N. Sharkey, "Automated Killers and the Computing Profession," Computer, vol. 40, no. 11, pp. 124-123, 2007.

[31] L. Takayama, W. Ju, and C. Nass, "Beyond dirty, dangerous and dull," in Proceedings of the 3rd international conference on Human robot interaction - HRI '08, Amsterdam, The Netherlands, 2008, p. 25.

[32] G. Aggarwal, E. Bursztein, C. Jackson, and D. Boneh, "An analysis of private browsing modes in modern browsers," in Proceedings of the 19th USENIX conference on Security, Berkeley, CA, USA, 2010, p. 6-6. 